\title{
Une nouvelle mention du dieu Mars Mullo : un graffite sur vase à Notre-Dame-du-Marillais (Le Marillais, Maine-et-Loire)
}

A new record of the god Mars Mullo: a graffito on a vase from Notre-Dame-duMarillais (Le Marillais, Maine-et-Loire)

François Bérard, Olivier Gabory, Martial Monteil, Christian Le Boulaire et Yves Saget

\section{OpenEdition Journals}

\author{
Édition électronique \\ URL : http://journals.openedition.org/rao/5709 \\ DOI : 10.4000/rao.5709 \\ ISBN : 978-2-7535-1608-3 \\ ISSN : 1775-3732 \\ Éditeur \\ Presses universitaires de Rennes \\ Édition imprimée \\ Date de publication : 20 décembre 2008 \\ Pagination : 261-268 \\ ISBN : 978-2-7535-0789-0 \\ ISSN : 0767-709X \\ Référence électronique \\ François Bérard, Olivier Gabory, Martial Monteil, Christian Le Boulaire et Yves Saget, « Une nouvelle \\ mention du dieu Mars Mullo : un graffite sur vase à Notre-Dame-du-Marillais (Le Marillais, Maine-et- \\ Loire) », Revue archéologique de l'Ouest [En ligne], 25 | 2008, mis en ligne le 30 mars 2020, consulté le \\ 04 décembre 2020. URL : http://journals.openedition.org/rao/5709 ; DOI : https://doi.org/10.4000/rao. \\ 5709
}




\title{
Une nouvelle mention du dieu Mars Mullo : un graffite sur vase à Notre-Dame-du-Marillais (Le Marillais, Maine-et-Loire)
}

\author{
A new record of the god Mars Mullo: a graffito on a vase from \\ Notre-Dame-du-Marillais (Le Marillais, Maine-et-Loire)
}

\author{
François BÉrarD*, Olivier Gabory** et Martial MonteiL ${ }^{* * *}$ \\ avec la collaboration de Christian Le Boulaire**** et Yves Saget ${ }^{* * * * *}$
}

\begin{abstract}
Résumé : Un bol en sigillée du Centre-Ouest, découvert fortuitement à Notre-Dame-du-Marillais (Maine-et-Loire), porte un graffite à caractère votif où sont mentionnés les puissances divines des Augustes et le dieu Mullo. Ce nouveau témoignage s'ajoute à un corpus d'une douzaine d'autres mentions de Mars Mullo, divinité poliade honorée dans plusieurs cités de l'Ouest de la Lyonnaise.

Abstract: A Terra Sigillata bowl from Central-West Gaul, discovered by chance on the site of Notre-Dame-du-Marillais (Maine-et-Loire, France), bears a votive graffito which mentions the divine spirits of the emperors and the god Mullo. This new evidence is to be added to a corpus of a dozen other records of Mars Mullo, poliade godhead honoured in several territories of the Western Lyonnaise province.
\end{abstract}

Mots clés : graffite, sigillée du Centre-Ouest, Haut-Empire, Mars Mullo.

Key words: graffite, Central-Western Gaul terra sigillata, High Empire, Mars Mullo.

\footnotetext{
* Professeur, Université Lyon 3, Faculté des Lettres et Civilisations, 7 rue Chevreul, 69007 Lyon; UMR 8546 «Archéologies d'Orient et d'Occident " (CNRS-ENS, Paris). (Francois.Berard@ens.fr)

** Directeur du Centre Permanent d'Initiatives pour l'Environnement Loire et Mauges, Maison de pays, BP 90025, 49601 Beaupreau Cedex. (cpie. loire-et-manges@paysdesmanges.fr)

*** Maître de Conférences, Université de Nantes, UFR Histoire, Histoire de l'Art et Archéologie, Chemin de la Censive du Tertre, BP 81227,44312 Nantes Cedex 3; UMR 6566 "Civilisations Atlantiques et Archéosciences" (Rennes). (Martial.monteil@univ-nantes.fr)

**** Archéologue et céramologue, Institut ational de Recherches archéologiques préventives (INRAP Grand-Ouest, 4 rue du Tertre, 44477 Carquefou Cedex. (Christian.le-boulaire@inrap.fr)

***** Archéologue bénévole, 55 rue de la Moisdonnière, 44300 Nantes.
} 


\section{ConteXte géographiQue ET HISTORIQUE DE LA DÉCOUVERTE}

\section{Le site de Notre-Dame-du-Marillais}

Le lieu de découverte - Notre-Dame-du-Marillais - doit son nom à un petit village, regroupé autour d'une basilique et localisé sur la commune du Marillais, dans la partie sud-ouest du Maine-et-Loire et à la limite avec la LoireAtlantique ${ }^{1}$. Il est localisé à environ $55 \mathrm{~km}$ à l'est de Nantes et à $45 \mathrm{~km}$ à l'ouest d'Angers (fig. 1).

Ce secteur, situé en rive gauche de la Loire, est particulièrement marqué par l'embouchure de l'Èvre, une rivière qui prend sa source sur la commune de Vezins à environ $50 \mathrm{~km}$ à vol d'oiseau, mais dont le parcours sinueux s'étale en réalité sur $97 \mathrm{~km}$. À son débouché dans la Loire, le lit de l'Èvre s'élargit notablement et s'écoule au travers d'alluvions récentes qui reposent sur un socle de schistes et de micaschistes. Ces alluvions sont, pour l'essentiel, le produit de crues successives de la Loire auxquelles se sont ajoutés, de manière plus réduite, des apports liés à la rivière affluente (Barre 1995).

Le cours d'eau est scandé par de multiples chaussées et biefs d'époques médiévale et moderne, destinés à alimenter des moulins pour la plupart abandonnés aujourd'hui. Au droit de Notre-Dame du Marillais et au contact de la Loire, il est marqué par la présence de plusieurs îles occupées par des prairies et des espaces boisés (fig. 2).

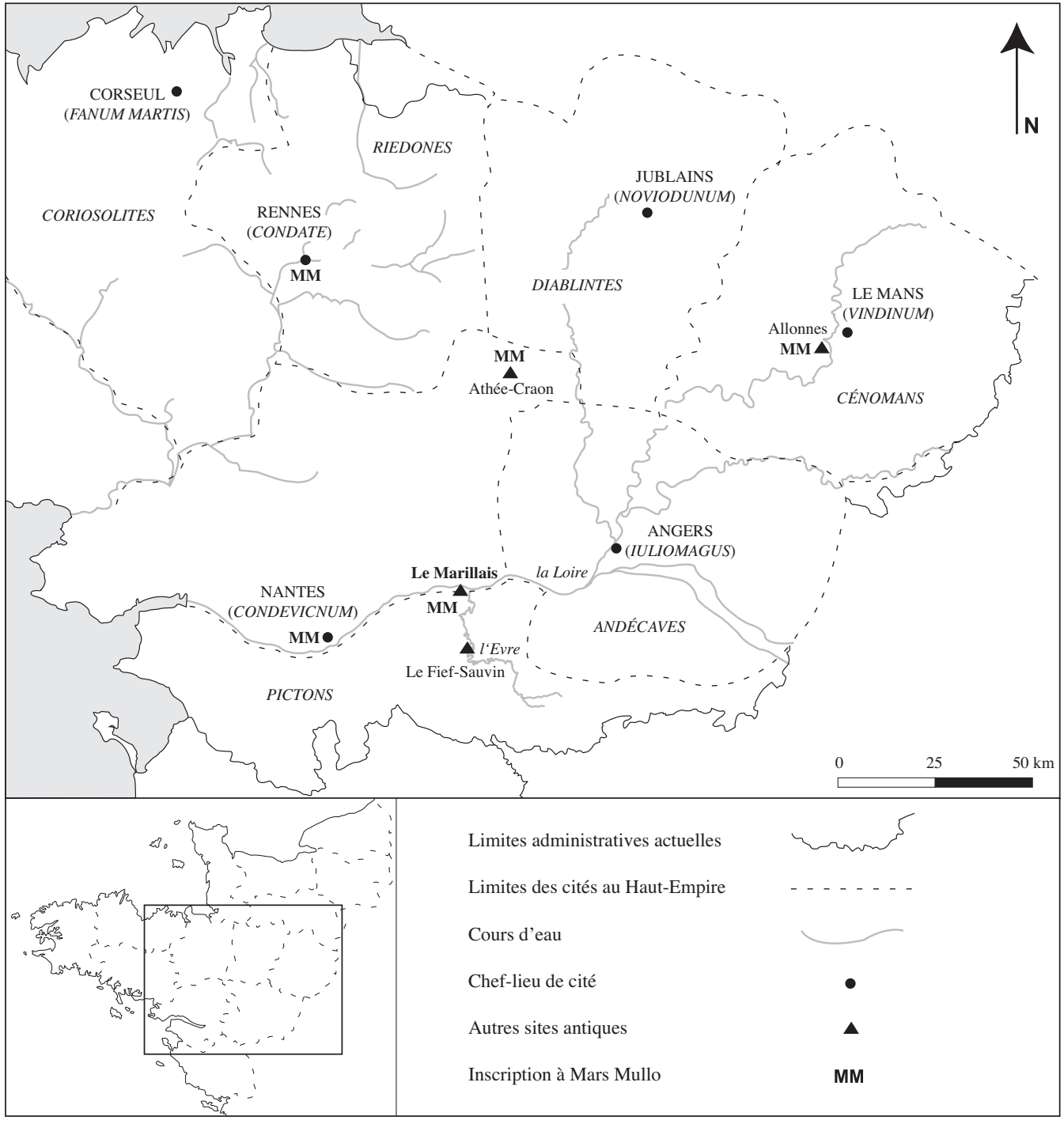

Figure 1 : Localisation du site de Notre-Dame-duMarillais (Maine-et-Loire) et des inscriptions mentionnant le dieu Mars Mullo dans le Grand-Ouest (réal. M. Monteil).

Figure 1: Location of NotreDame-du-Marillais site (Maine-et-Loire) and of the inscriptions mentionning god Mars Mullo in the west of France.

1. La découverte dont il est question dans cet article a fait l'objet d'une première présentation en 2007 (Bérard et al., 2007). 


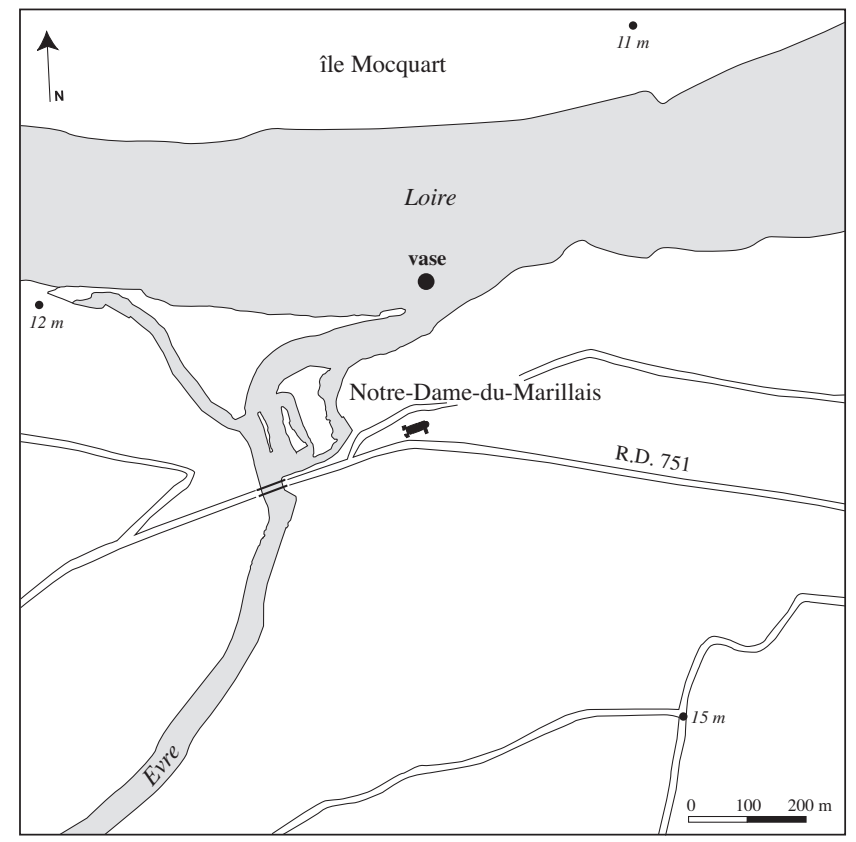

Figure 2 : Carte générale du site de Notre-Dame-du-Marillais et du point de découverte du vase avec graffite (réal. M. Monteil). Figure 2: General map of Notre-Dame-du-Marillais site with the discovery place of the inscribed vase.

\section{Environnement archéologique}

L'agglomération antique la plus proche est celle du FiefSauvin (La Ségourie), souvent assimilée au Segora de la table de Peutinger et située à $16 \mathrm{~km}$ au sud (Provost, 1988a, p. 75). Plus largement, Le Marillais est intégré au pays dit des Mauges; ce dernier fait partie, avec le pays de Retz voisin, d'un territoire que plusieurs chercheurs ont assimilé à celui du peuple des Ambiliates évoqué notamment par César (Guerre des Gaules, III, 9), mais aussi, sous la forme Ambilatres, par Pline l'Ancien (IV, 108, 1-4) dans sa liste des peuples de l'Aquitaine. Ce petit peuple du sud de la Loire aurait été rattaché, sous César ou Auguste, aux Pictons pour remercier ces derniers de leur soutien dans la guerre contre les Vénètes et pour leur ouvrir les portes du commerce ligérien (Hiernard, 1979, p. 61-64; voir aussi Provost, 1993, p. 87-88 et Berthaud [dir.], 2000, p. 17-18). En dernier lieu, A. Levillayer a bien montré, dans le cadre d'un mémoire de maîtrise, que ce petit territoire a occupé une position de carrefour tout au long de l'Antiquité et du Moyen Âge (Levillayer, 2003). Dans l'Antiquité, la zone de découverte est ainsi placée au contact de trois cités : celle des Andécaves (chef-lieu de cité Angers), celle des Namnètes (Nantes) et celle des Pictons (Poitiers).

Les découvertes archéologiques d'époque antique faites sur la commune du Marillais et sur celle, voisine, de Saint-
Florent-le-Vieil, sont peu nombreuses et se résumaient, il y a peu, à une tombe à crémation secondaire datée du $\mathrm{III}^{\mathrm{e}}$ ou du IV siècle de notre ère (Monteil, 2004, p. 117-118) et à quelques monnaies et objets isolés (Provost, 1988a, p. 75).

Le site de Notre-Dame-du-Marillais a été révélé en 1991 suite à la découverte fortuite d'une amphore quasi complète de type Dressel 20, frâichement brisée par un engin de chantier. Régulièrement érodé par les crues de l'Èvre et de la Loire, il livre chaque année des centaines de fragments de céramique, peu éloignés de leur situation d'origine si l'on en juge par leur état de fraîcheur et leurs cassures récentes. Des chercheurs amateurs locaux, membres de l'association RABLE (Recherches archéologiques dans le Bassin de la Loire et de l'Èvre), y effectuent, depuis plus de quinze ans et à des fins de sauvetage, un patient travail de ramassage et d'inventaire des objets mis au jour durant les périodes d'étiage. C'est dans ce cadre que le vase complet dont il est question ici a été découvert par Georges Trichet (fig. 2).

Le contexte d'origine des milliers de tessons de vases antiques ainsi recueillis hors stratigraphie reste encore mal connu, mais correspond manifestement à un site important. En 2004, C. Le Boulaire a effectué une première analyse de la collection dans le cadre de recherches coordonnées par A. de Saulce (Service régional de l'Archéologie des Pays de la Loire) (Le Boulaire, 2004). Cette étude a été centrée sur la datation (450 tessons examinés) et sur la recherche d'anomalies susceptibles d'aider à l'interprétation du gisement. Le mobilier examiné est marqué par des distorsions liées aux conditions de ramassage, mais s'insère globalement entre les ${ }^{\text {er }}$ et $\mathrm{VII}^{\mathrm{e}}$ siècles de notre ère, avec un pic majoritaire pour le Haut-Empire. Sa composition est celle d'un vaisselier «classique " dans un cadre d'habitat, mais ce dernier est manifestement en lien étroit avec les principaux courants commerciaux, notamment durant l'Antiquité tardive (sigillée d'Argonne; dérivée de sigillée paléochrétienne).

Plus récemment, en 2006, une équipe d'archéologues de l'INRAP et du RABLE a procédé à une série de sondages sur le site, avec l'espoir d'y reconnaître des vestiges bâtis. Cette enquête a livré des quantités importantes de mobilier incluses dans des dépôts alluvionnaires de l'Èvre, mais n'a pas permis de localiser leur lieu d'origine (Fillon et Viau, 2007).

\section{Description du Vase et DU GRAFFite}

Le vase en céramique objet de cette note, complet, est un grand bol à collerette en sigillée de type Drag. 38 cuit en mode A (diam. à l'ouverture $20 \mathrm{~cm}$, hauteur 10,5 cm) (fig. 3 et 4). Sa pâte, micacée, est de teinte gris bistre à brune en surface. L'engobe, non grésé, est de couleur brun rouge et a 


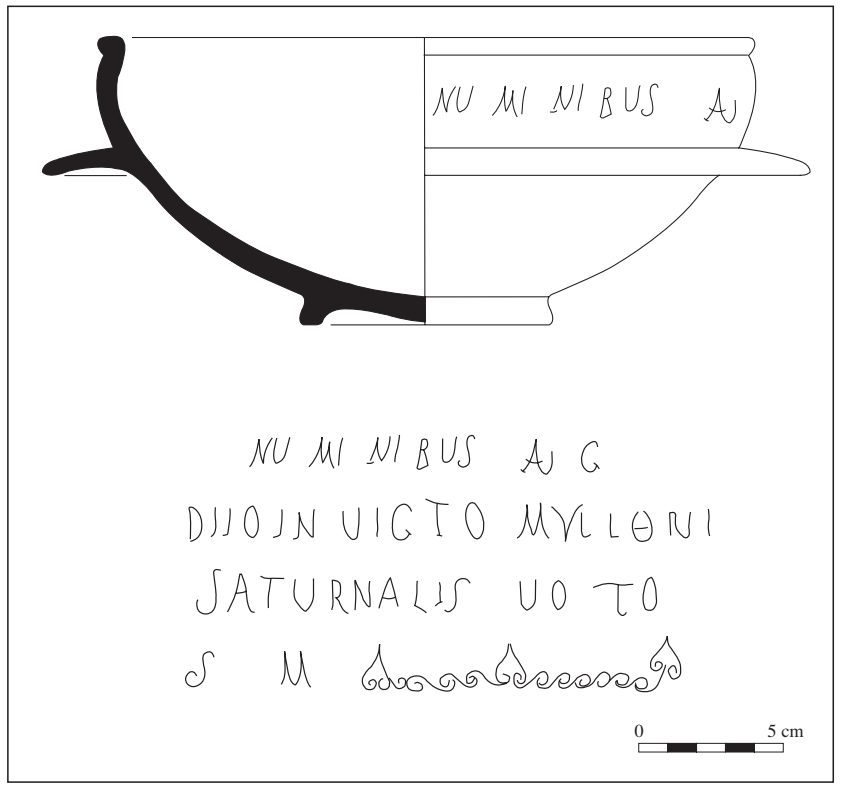

Figure 3 : Dessin du vase et de l'inscription (réal. M. Monteil, J. Mornand, Y. Saget).

Figure 3: Drawing of vase and inscription.

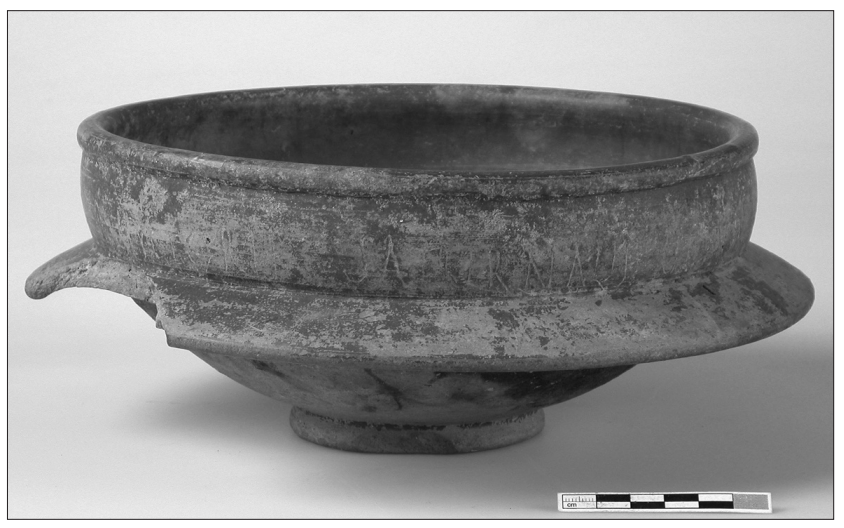

Figure $4:$ Le vase vu de face : on distingue sur le bandeau une partie du graffite ... MULLONI SATURNALIS (Cl. J.-G. Aubert, Arc'Antique).

Figure 4: Front view of the vase seen by face: a part of the graffito (...MULLONI SATURNALIS) is visible.

quasiment disparu. L'intérieur est extrêmement usé sur plus de la moitié de la hauteur et l'extérieur présente des traces de chauffe. La panse présente en un point une perforation d'environ $2 \mathrm{~cm}^{2}$, pratiquée depuis l'extérieur vers l'intérieur et qui parait bien résulter d'un acte volontaire (fig. 5). Mis à part cet indice d'une forme de sacrifice bien connue en contexte cultuel ou funéraire, il s'agit là d'un vase qui fait partie de la vaisselle de table ordinaire, où il sert à la présentation des aliments liquides ou solides.

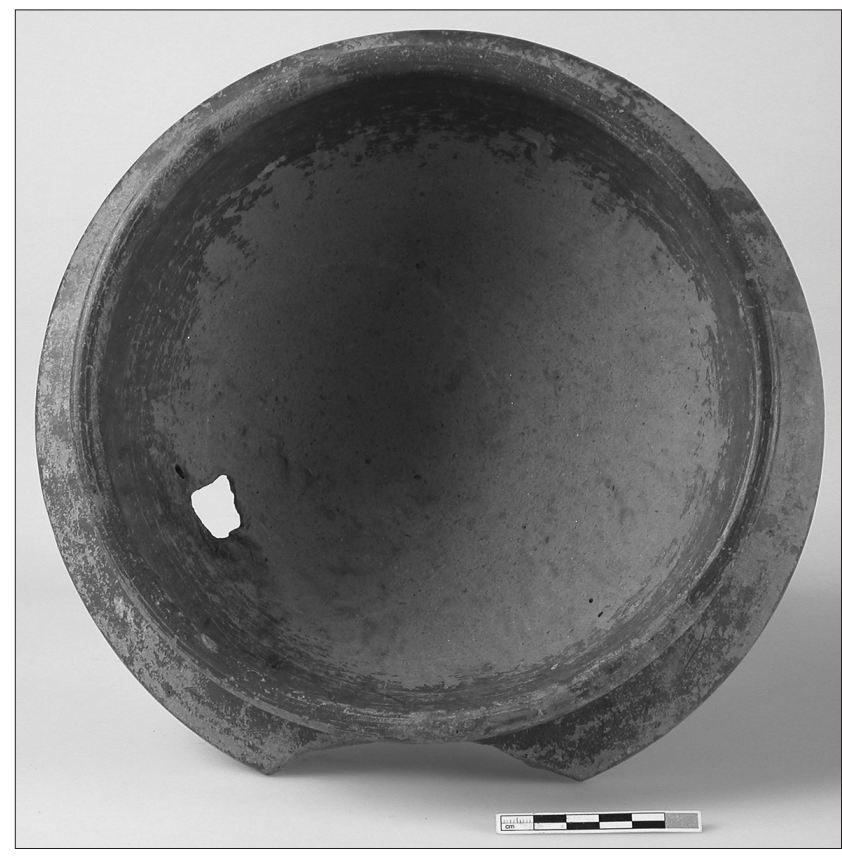

Figure 5 : Vue de l'intérieur du vase montrant les traces d'usure et une perforation volontaire (Cl. J.-G. Aubert, Arc'Antique).

Figure 5: Interior of the vase showing traces of wear and a deliberate perforation.

La texture de la pâte est caractéristique d'une production issue des ateliers de sigillée du Centre-Ouest ${ }^{2}$. Ces derniers, qui ne sont pas reconnus avec certitude, doivent sans doute être localisés dans les vallées de la Vienne et du Clain, ainsi que dans la région de Cholet. Leur production est principalement diffusée dans l'ouest de la Gaule, entre la fin du $\mathrm{I}^{\mathrm{er}}$ siècle et le milieu du $\mathrm{II}^{\mathrm{e}}$. Ces sigillées se retrouvent notamment en Pays de la Loire et en Bretagne (Delage, 1997), et en quantité assez importante dans l'estuaire de la Loire. Là, le bol de forme Drag. 38, encore assez rare à la fin du I $\mathrm{I}^{\mathrm{er}}$ siècle, est plus fréquemment attesté dans la première moitié du II ${ }^{\mathrm{e}}$. (Guitton, 2004).

Le bandeau du bol porte une inscription gravée à la pointe sèche après cuisson qui se développe sur tout le pourtour et s'achève sur un rinceau de $S$ à deux volutes, ponctué par trois palmettes stylisées (fig. 3 et 6). Les lettres sont tracées en capitales, vraisemblablement par un droitier, et sont hautes en moyenne de 1 à $1,2 \mathrm{~cm}$ : NVMINIBVS AVG(ustorum) DEO INVICTO MVLLONI SATVRNALIS VOTO S(oluto) $M($ erito), ce qui peut être traduit par : «Aux puissances divines des Augustes et au Dieu invincible Mullo, Saturnalis, en accomplissement de son vœu et à juste titre. »

2. Nous remercions David Guitton (Inrap) qui a confirmé l'identification préliminaire proposée par Christian Le Boulaire. 

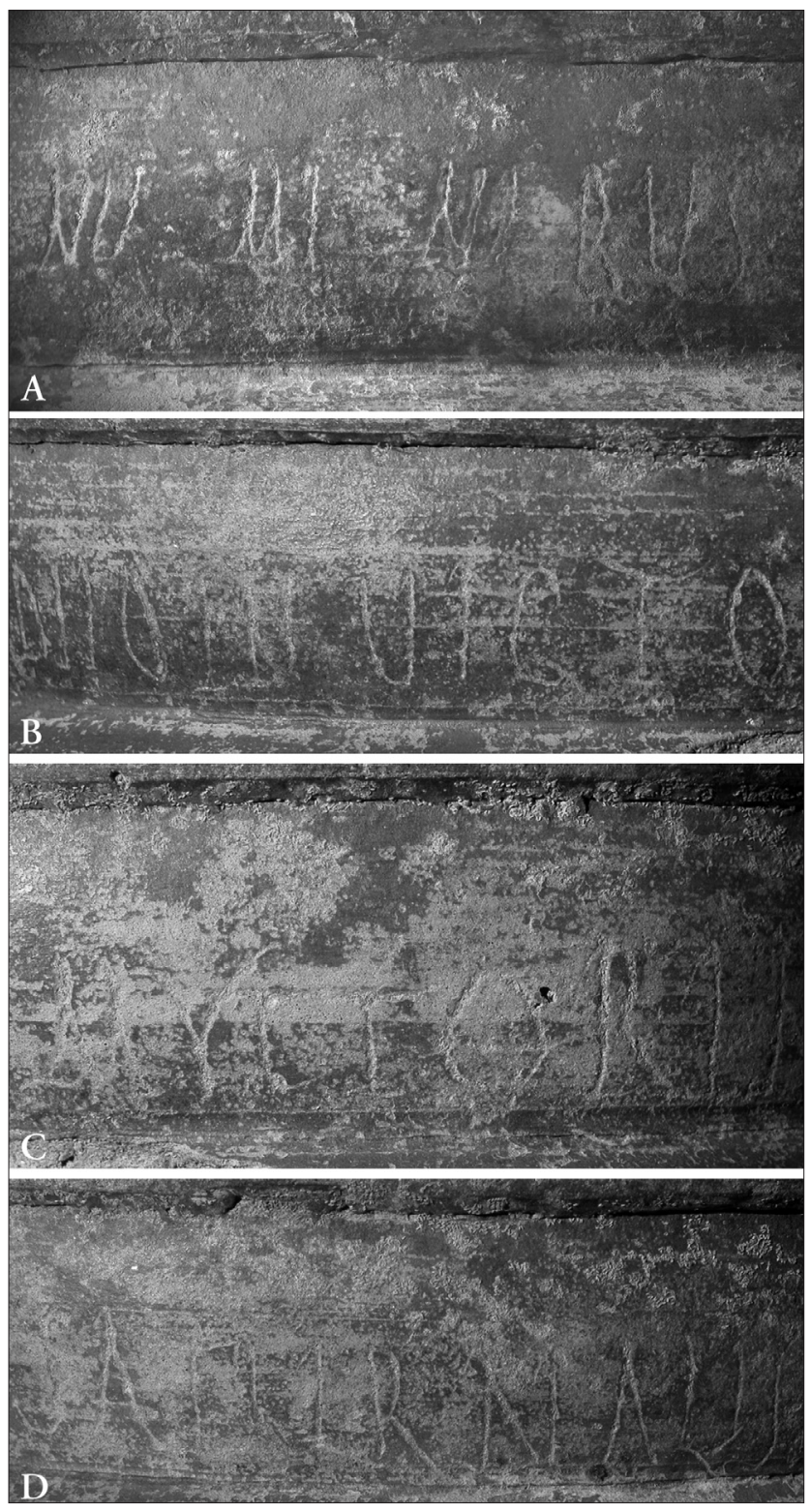

Figure 6 : Détails de l'inscription : A, NUMINIBUS; B, DEO INVICTO; C, MULLONI; D, SATURNALIS (Cl. J.-G. Aubert, Arc'Antique).

Figure 6: Details of the inscription.

Ce graffite correspond à un acte religieux, en l'occurrence une offrande (ici le vase) faite aux divinités en acquittement (solutio) d'un vou (votum). La formule employée en fin d'inscription, sans être rare, est moins fréquente que celle qui est abrégée en VSLM - v(otum) s(olvit) l(ibens) m(erito) - et qui se traduit par: " untel a accompli son vœu de bon gré et à juste titre".

Cette dédicace associe culte impérial et culte d'une divinité régionale comme l'indique la mention des puissances divines des Augustes et du dieu invincible Mullo. Ce type d'association est très fréquent à partir du règne d'Hadrien, à l'échelle des Trois Gaules et des Germanies ou encore de la Narbonnaise et, comme à l'ordinaire, la mention des numina impériaux vient avant celle du dieu. Sans chercher bien loin, on remarquera que ce formulaire est par exemple attesté à Nantes avec le dieu Vulcain (CIL XIII, 3106 et 3107) ou encore avec le dieu Mars (CIL XIII, 3103; Provost, 1988b, p. 95). Dans le cas présent, les termes Numinibus Aug(ustorum) indiquent une dédicace faite à l'ensemble des empereurs vivants et morts, mais d'autres formules semblables, plus ou moins abrégées (Num. Aug., Numin. Aug., etc.), prêtent davantage à discussion (Fishwick, 1991, p. 388-396; Raepsaet-Charlier, 1993, p. 43-47; Chastagnol, 1999, p. 78; Van Andringa, 2002, p. 167-170, 173-174; Cibu, 2003, p. 344-345).

Sur toutes les inscriptions gravées sur pierre attestées dans l'Ouest, le théonyme Mullo est systématiquement associé à celui de Mars. Ici, il est réduit à sa seule forme indigène, tout comme dans le graffite sur peinture murale découvert dans le sanctuaire des Provenchères à Athée-Craon (Mayenne), si ce dernier est bien complet (Naveau, 2000, p. 5, fig. 2). Il est toutefois précédé de deo (dieu), dans une association qui est là encore courante dans les Gaules (Raepsaet-Charlier, 1993, p. 12-17; Van Andringa, 2002, p. 134; Cibu, 2003, p. 345-348), et il est suivi de l'épithète invicto (invincible), assez répandue comme son équivalent victor (victorieux). On la trouve pour d'autres dieux, comme Hercule, Sérapis et surtout bien sûr Mithra. Mais elle n'est pas inconnue pour Mars qui, sous le nom d'Invictus, avait à Rome un temple (LTUR, III, 229-230) et une fête, le 14 mai (Fast. Ven. = Inscr. Italiae, XIII, 2, p. $57 ; c f .457)^{3}$. Le nom du dédicant enfin, forgé sur celui du dieu Saturne ou des Saturnales, n'est pas fréquent (Kajanto, 1965, p. 54 et 220) : on n'en connaît qu'une attestation en Gaule Lyonnaise, à Sens (Yonne), où une stèle datée de la première moitié du II ${ }^{\mathrm{e}}$ siècle porte la mention Saturnalis, fils de Saturninus (CIL XIII, 2990).

La datation doit prendre en compte plusieurs critères. Le support lui-même, tout d'abord, est un vase en sigillée du Centre-Ouest dont la production s'inscrit entre l'extrême fin du i ${ }^{\text {er }}$ siècle et le milieu du $\mathrm{II}^{\mathrm{e}}$. Il est fortement usé, ce qui indique une utilisation prolongée. Dans le graffite, ensuite, la mention deo, placée devant le nom de la divinité, est en général datée postérieurement au règne d'Hadrien et est surtout utilisée entre le milieu du $\mathrm{II}^{\mathrm{e}}$ et le milieu du $\mathrm{III}^{\mathrm{e}}$ siècle (Raepsaet-Charlier, 1993, p. 12-17; Cibu, 2003, p. 345348). Une datation située dans le courant de la seconde moitié du $\mathrm{II}^{\mathrm{e}}$ siècle ou de la première moitié du $\mathrm{III}^{\mathrm{e}}$ pourrait donc convenir.

3. Cf. aussi CIL VI, 33856, à Rome, et XIII, 392, à Pouzac (HautesPyrénées), dans la province d'Aquitaine. 


\section{Mars Mullo, une divinité poliade DE LA LYONNAISE OCCIDENTALE}

Le dossier de ce Mars indigène - dont il n'est pas question de donner le détail ici - a été repris récemment (Naveau, 2000 ; Van Andringa, 2002, p. 141-144; Bérard, 2006). Il montre qu'une divinité indigène (Mullo), assimilée à un dieu romain (Mars), a occupé une place particulière à l'échelle de plusieurs cités de la partie occidentale de la province de Lyonnaise, en s'affirmant comme un grand dieu public, une divinité tutélaire ou poliade.

On a cherché à traduire l'épithète indigène de ce dieu en en faisant celui des muletiers (Mowat, 1879, p. 253-254), liés au transport de l'étain (Hiernard, 1982, p. 535-537), ou tout récemment celui des mines d'or (Pailler, 2006). Mais plutôt qu'une épithète, ne peut-on voir plus simplement dans Mullo le nom du dieu gaulois, sans y chercher nécessairement une fonction précise? (Naveau, 2000, p. 26-27).

Le corpus épigraphique qui concerne cette divinité est riche d'une douzaine de mentions, fait exceptionnel à l'échelle d'une région où les inscriptions sont relativement rares. Il est ainsi mentionné cinq fois à Rennes (RouanetLiesenfelt, 1980, p. 20-29; Leroux et Provost, 1990, p. 192$193)$ : sur quatre bases de statues (CIL XIII, 3148 et 3149 ; $A E$ 1967-1970, 405b; CIL XIII, 3151 = AE 1969-1970, $405 c)$ et sur une inscription honorifique ( $A E$ 1969-1970, 405a). À Nantes (Provost 1988b), il est sûrement évoqué en deux occasions (CIL XIII, 3101 et 3102) et peut-être une troisième fois (CIL XIII, 3103). À Allonnes (Sarthe), le grand sanctuaire de Mars Mullo est associé à trois mentions de ce dieu (ILTG, 343, 344 et 345; Brouquier-Reddé et Gruel (dir.), 2004, p. 308-310). Enfin, l'agglomération antique d'Athée-Craon (Mayenne) et son sanctuaire dit des Provenchères ont fourni deux autres témoignages, l'un sur pierre (CIL XIII, 3096) et l'autre sous la forme d'un graffite sur enduit mural (CIL XIII, 3097i; Naveau, 2000, p. 4-8).

Ce culte est donc attesté chez les Riédons, les Aulerques Cénomans et les Namnètes, la petite région du Craonnais dépendant de ce dernier peuple et non de celui des Diablintes si l'on suit J.-C. Meuret (1993, p. 255-260). Comme le souligne Van Andringa, "l'homogénéité d'une telle répartition est sans doute le souvenir de liens ethniques ou politiques qui existaient entre ces divers peuples depuis une époque ancienne, présidés par une même divinité Mullo » (Van Andringa, 2002, p. 141-142).

Le graffite de Notre-Dame-du-Marillais vient donc compléter une série déjà importante et nous livre quelques informations supplémentaires.

Tout d'abord, ce graffite votif sur vase constitue, à notre connaissance, un unicum à l'échelle des régions de Bretagne et Pays de la Loire. Les graffites sur céramiques publiés à ce jour correspondent en effet à des indications de contenu, à des marques de propriété, de volume ou encore de poids, mais aucun ne renvoie à un acte cultuel semblable à celui qui est illustré ici. Il en est ainsi en Pays de la Loire, si l'on excepte une assimilation peu convaincante entre un graffite $E s u$, inscrit sur au moins trois vases de la nécropole de Jublains (Mayenne), et le dieu gaulois Esus (Demeslay, 1988, p. 49). Le fait est confirmé dans la cité des Aulerques Cénomans, bien documentée grâce aux fouilles du sanctuaire de Mars Mullo à Allonnes et à un répertoire de graffites récemment édité (Guillier, Thauré, 2003). En Bretagne, malgré l'absence de recensement exhaustif récent, les données disponibles ne fournissent pas non plus d'exemples semblables à celui du Marillais.

Dans ces deux régions, les seuls graffites à caractère votif rencontrés sur instrumentum semblent bien être ceux inscrits sur les vases en argent du trésor de Notre-Dame-d'Allençon (Maine-et-Loire), qui font référence à Minerve (CIL XIII, 3100/1-16; Baratte, 1981).

Dans un cadre géographique plus large, on signalera seulement une inscription portée elle aussi sur le bord d'un vase en céramique rouge, découvert dans les années 1930 dans la région de Beaune et qui paraît constituer un bon parallèle pour celle du Marillais : Deo invicto Mytrae uassa decem Tertius Rustici (flius) u(otum) s(oluit) l(ibens) l(aetus) $m$ (erito) $(A E, 1933,240)$ et, plus récemment, un ensemble de plusieurs plats ou gobelets découverts dans le mithraeum de Martigny en Suisse et dont deux sont dédiés deo invicto $\operatorname{Mitr}($ ae) (AE, 1998, 868-869; Wiblé, 1995).

La dénomination du dieu Mullo, sans le nom romain de Mars, serait, comme dans le graffite du temple des Provenchères à Athée-Craon, un témoignage de la dévotion populaire qui attache sans doute moins d'importance à la version officielle (Mars Mullo) qu'à la dénomination traditionnelle (Naveau, 2000, p. 5). Le qualificatif d'invictus (invaincu, ou plutôt invincible) qui lui est associé est inédit dans la série épigraphique : il renvoie au caractère guerrier du dieu, également attesté - parmi d'autres aspects qui attestent de sa polyvalence - par l'analyse du décor architectural du sanctuaire d'Allonnes (Brouquier-Reddé et Gruel [dir.], 2004, p. 357-360).

L'association d'une divinité locale avec les puissances divines des Augustes, est en revanche un phénomène très courant dans l'épigraphie de la Gaule romaine, qui confirme le caractère public et officiel du culte.

\section{Conclusion}

Cette découverte, assez exceptionnelle dans une région où les inscriptions antiques sont rares, vient donc compléter le 
corpus des mentions du dieu Mars Mullo. L'analyse ne peut guère être poussée au-delà de la présentation de cet " objet parlant " qu'est le vase inscrit (Rey-Vodoz, 2006, p. 222), faute de renseignements sur son contexte d'origine. Les sondages réalisés dans ce secteur en 2006 n'ont en effet pas permis de localiser l'emplacement précis des vestiges antiques, mais ont toutefois prouvé que son érosion était imputable à l'Èvre et non à la Loire. C'est donc sur les rives de ce cours d'eau, dont le tracé a dû fluctuer avec le temps, qu'il convient de le rechercher. Les quantités de mobilier recueillies ces dernières années attestent en tout cas l'importance du gisement, qui pourrait correspondre à un sanctuaire avec bâtiments annexes, à une villa ou à une agglomération.

Quoi qu'il en soit, il n'est pas interdit de noter que ce graffite étend a priori l'extension géographique de la divinité Mars Mullo à la rive gauche de la Loire, et donc au nord de la cité des Pictons si l'on s'en tient aux restitutions cartographiques les plus récentes (par exemple Berthaud [dir.], 2000, fig. 5). Il convient évidemment de rester prudent car le vase pourrait aussi bien correspondre à une offrande isolée, déposée par un voyageur dans un sanctuaire à caractère polythéiste, ou même à un objet récupéré ailleurs. Mais cette découverte pourrait aussi constituer, à titre d'hypothèse, un argument pour restituer dans ce secteur une population attachée à des valeurs religieuses partagées avec les Riédons, les Aulerques Cénomans et les Namnètes.

\section{Bibliographie}

$A E-L$ Lannée épigraphique, Paris.

BARATte, F., 1981 - Le trésor d'orfévrerie gallo-romaine de NotreDame d'Alençon (Maine-et-Loire), Paris, CNRS, (Supplément à Gallia, 40), $90 \mathrm{p}$.

BARRE, O, 1995 - Contribution à la connaissance de l'Evvre et de son bassin. Étude de géographie, (Maîtrise de Géographie), Université d'Angers.

Bérard, F., 2006 - Mars Mullo : un Mars des cités occidentales de la province de Lyonnaise, in Brouquier-Reddé, V., et al. (éd.), Mars en Occident (Actes du colloque international "Autour d'Allonnes (Sarthe), les sanctuaires de Mars en Occident », Le Mans, 2003), Rennes, Presses Universitaires de Rennes, p. 17-33.

Berard, F., Gabory, O. et Monteil, M. (avec la collaboration de Le Boulaire, C. et SAGEt Y., 2007) - Une nouvelle mention du dieu Mars Mullo : un graffite sur vase au Marillais (Maineet-Loire), in de Saulce, A., Serna, V. et Gallicé, A. (dir.), Archéologie en Loire. Actualité de la recherche dans les régions Centre et Pays de la Loire. (Aestuaria 12), Cordemais, éditions Estuarium, p. 365-373.
Berthaud, G., (dir.) 2000 - Mazières-en-Mauges gallo-romain (Maine-et-Loire). Un quartier à vocation artisanale et domestique, Angers, ARDA, 262 p.

Brouquier-Reddé, V. et Gruel, K. (dir.), 2004 - Le sanctuaire de Mars Mullo chez les Aulerques Cénomans (Allonnes, Sarthe). $V^{e}$ s. av. J.-C. - IV ${ }^{\mathrm{e}}$ s. apr. J.-C. État des recherches actuelles. Gallia, 61, p. 291-396.

César - Guerre des Gaules, texte établi, traduit et commenté par Constant, A.-L., 1950, Paris, Les Belles Lettres.

Chastagnol, A., 1999 - Réflexions sur l'évolution du formulaire épigraphique dans les dédicaces locales du culte impérial. Revue archéologique de Narbonnaise, 32, p. 5-10.

Cibu, S., 2003 - Chronologie et formulaire dans les inscriptions religieuses de Narbonnaise et des provinces alpines. Revue archéologique de Narbonnaise, 36, p. 335-360.

CIL $\boldsymbol{V I}$ = Huelsen, Chr., 1902 - Corpus Inscriptionum Latinarum, VI, Inscriptiones Urbis Romae Latinae, IV/2, Berlin.

CIL XIII = Hirschfeld, O., 1899 - Corpus Inscriptionum Latinarum, XIII, 1.1, Inscriptiones Aquitaniae et Lugdunensis, Berlin.

Delage, R., 1997 - Premier aperçu de la diffusion de la céramique sigillée du groupe Centre-Ouest dans l'ouest de la Gaule (régions Pays de la Loire et Bretagne) - SFECAG, Actes du congrès du Mans, 1997, Marseille, SFECAG, p. 279-292.

Demeslay, F., 1988 - Les Graffites sur céramique à l'époque galloromaine dans les Pays de Loire (Maîtrise d'Histoire), Université de Nantes.

Fillon, D. ET Viau, Y., 2007 - Le site de Notre-Dame-du-Marillais (Maine-et-Loire), in De Saulce, A., Serna, V. et Gallicé, A. (dir.), Archéologie en Loire. Actualité de la recherche dans les régions Centre et Pays de la Loire. (Aestuaria 12), Cordemais, éditions Estuarium, p. 361-364.

Fisнwicк, D., 1991 - The Imperial Cult in the Latin West, II/1, Leiden.

Guillier, G. et Thauré, M., 2003 - Les graffites antiques de la cité des Aulerques Cénomans, Montagnac, Éditions Monique Mergoil (Monographies Instrumentum, 25), 298 p.

Guitton, D., 2004 - Contribution à l'étude de la diffusion des céramiques sigillées du groupe Centre-Ouest : l'estuaire de la Loire. SFECAG. Actes du congrès de Vallauris, 2004, Marseille, SFECAG, p. 289-314.

ILTG = WuIlleumier, P., 1963 - Inscriptions latines des Trois Gaules, Paris, CNRS, (Gallia, supplément 17).

Hiernard, J., 1979 - Poitou et Vendée avant les Romains : une enquête numismatique, Annuaire Vendée, 126, p. 45-111.

Hiernard, J., 1982 - Corbilo et la route de l'étain, Bulletin de la Société des Antiquaires de l'Ouest et des musées de Poitiers, $4^{\mathrm{e}}$ série, 16, p. 497-578.

Kajanto, I., 1965 - The Latin Cognomina, Helsinki, 417 p.

Le Boulaire, C., 2004 - La céramique de Notre-Dame-duMarillais, in DE SAulce, A. (dir.), Rapport de Programme col- 
lectif de Recherche sur la Loire et ses affluents, Nantes, Service régional de l'Archéologie des Pays de la Loire, p. 35-49.

Leroux, G. et Provost, A., 1990 - Carte archéologique de la Gaule, L'Ille-et-Vilaine 35, Paris, Académie des Inscriptions et Belles-Lettres, $304 \mathrm{p}$.

Levillayer, A., 2003 - Les Mauges (Maine-et-Loire, LoireAtlantique, Vendée) : archéologie d'un terroir du premier âge du Fer au début du haut Moyen Âge (Maîtrise d'archéologie), Université de Rennes 2, 2 volumes.

$\boldsymbol{L T U R}=$ STEINBY E.-M. (dir.), 1996 - Lexicon Topographicum urbis Romae, III, Roma, Quasar, 504 p.

Meuret, J.-Cl., 1993 - Peuplement, pouvoir et paysage sur la marche Anjou-Bretagne, La Mayenne: Archéologie, Histoire, suppl. 4, 656 p.

MonteIL, M., 2004 - Tombes et nécropoles rurales à incinération du Haut-Empire. Un état des recherches en Pays de la Loire. Bulletin de la Société archéologique et historique de la LoireAtlantique, 139, p. 93-139.

Mowat, R., 1879 - Remarques sur les inscriptions antiques du Maine. II, Département de la Mayenne, $\mathrm{n}^{\circ} 8$, Congrès Archéologique de France, (45 session, Le Mans-Laval, 1878), Paris, p. 248-271.

Naveau, J., 2000 - Mullo, un dieu dans l'ouest de la Gaule. La Mayenne: Archéologie, Histoire, 23, p. 3-33.

Pailler, J.-M., 2006 - Quand l'argent était d'or. Paroles de Gaulois. Gallia, 63, p. 211-241.
Pune L'AnCIEN - Histoire Naturelle, IV.

Provost, M, 1988a - Carte archéologique de la Gaule, Maine-etLoire 49, Paris, Académie des Inscriptions et Belles-Lettres, $174 \mathrm{p}$.

Provost, M, 1988b - Carte archéologique de la Gaule, LoireAtlantique 44, Paris, Académie des Inscriptions et BellesLettres, $177 \mathrm{p}$.

Provost, M., 1993 - Le Val de Loire dans l'Antiquité, Paris, CNRS (Supplément à Gallia, 52), 425 p.

Raepsaet-Charlier, M.-Th., 1993 - Diis deabusque sacrum. Formulaire votif et datation dans les Trois Gaules et les deux Germanies, Paris, de Boccard, 95 p.

Rey-Vodoz, V., 2006 - Offrandes et rituels votifs dans les sanctuaires de Gaule romaine, in Dondin-Payre, M. et RaepsaetCharlier, M.-Th. (éd.), Sanctuaires, pratiques cultuelles et territoires civiques dans l'Occident romain, Bruxelles, Le Livre Timperman, p. 219-258.

Rouanet-Liesenfelt, A.-M., 1980 - La civilisation des Riedones, Archéologie en Bretagne, suppl. 2, 297 p.

VAn Andringa, W., 2002 - La religion en Gaule romaine. Piété et politique (Ir-IIt siècles après J.-C.), Paris, Errance, 335 p.

Wiblé, F., 1995 - Le mithraeum de Forum Claudii Vallensium/ Martigny (Valais), Archäologie der Schweiz, 18/1, p. 2-15. 